\title{
Cyclotron emission, absorption, and the two faces of X-ray pulsar A $0535+262$
}

\author{
Sergey S. Tsygankov, ${ }^{1,2 \star ~ V i c t o r ~ D o r o s h e n k o, ~}{ }^{3,2}$ Alexander A. Mushtukov, ${ }^{4,2,5}$ \\ Valery F. Suleimanov, ${ }^{3,6,2}$ Alexander A. Lutovinov, ${ }^{2,7}$ and Juri Poutanen ${ }^{1,2}$ \\ ${ }^{1}$ Department of Physics and Astronomy, FI-20014 University of Turku, Finland \\ ${ }^{2}$ Space Research Institute of the Russian Academy of Sciences, Profsoyuznaya Str. 84/32, Moscow 117997, Russia \\ ${ }^{3}$ Institut für Astronomie und Astrophysik, Universität Tübingen, Sand 1, D-72076 Tübingen, Germany \\ ${ }^{4}$ Leiden Observatory, Leiden University, NL-2300RA Leiden, The Netherlands \\ ${ }^{5}$ Pulkovo Observatory, Russian Academy of Sciences, Saint Petersburg 196140, Russia \\ ${ }^{6}$ Kazan (Volga region) Federal University, Kremlevskaya str. 18, 420008 Kazan, Russia \\ ${ }^{7}$ Higher School of Economics, Myasnitskaya 20, 101000 Moscow, Russia
}

Accepted 2019 May 22. Received 2019 May 22; in original form 2019 April 3

\begin{abstract}
Deep NuSTAR observation of X-ray pulsar A $0535+262$, performed at a very low luminosity of $\sim 7 \times 10^{34} \mathrm{erg} \mathrm{s}^{-1}$, revealed the presence of two spectral components. We argue that the high-energy component is associated with cyclotron emission from recombination of electrons collisionally excited to the upper Landau levels. The cyclotron line energy of $E_{\mathrm{cyc}}=47.7 \pm 0.8 \mathrm{keV}$ was measured at the luminosity of almost an order of magnitude lower than what was achieved before. The data firmly exclude a positive correlation of the cyclotron energy with the mass accretion rate in this source.
\end{abstract}

Key words: accretion, accretion discs - pulsars: general - scattering - stars: magnetic field - stars: neutron - X-rays: binaries

\section{INTRODUCTION}

Physical processes dominating the X-ray emission from accreting neutron stars (NSs) depend strongly on the properties of the NS and the mass accretion rate. X-ray pulsars (XRPs), constituting a separate subclass of accreting NSs, are especially interesting in this respect allowing us to study microphysics under extreme conditions of ultrastrong magnetic fields. Observations of such systems at very low luminosities are of particular interest as they reveal the emission region in its simplest form of the hotspot at the NS surface, allowing us to avoid modeling of several complex effects associated with potential formation of an accretion column, propagation of light from the column to the observer, and illumination of the NS surface. However, even in this case the nature brought some surprises. In particular, such observations revealed dramatic transition from a cut-off power-law spectrum typical for XRPs at high luminosities to a two-component spectrum with peaks around 5 and $40 \mathrm{keV}$ at an accretion rate as low as $\sim 10^{14} \mathrm{~g} \mathrm{~s}^{-1}$ in a transient pulsar GX 304-1 (Tsygankov et al. 2019). Similarly to another low-luminosity XRP, X Persei, such spectral shape cannot be explained with the existing models devel-

* E-mail: sergey.tsygankov@utu.fi oped for high accretion rate sources (see e.g. Becker \& Wolff 2007; Farinelli et al. 2016).

At very low rates the emission is produced at the NS surface and allow to study the interaction of the infalling material with the highly magnetized atmosphere. Several mechanisms are able to explain the existence of the high-energy spectral component, with the most plausible being (i) the cyclotron emission reprocessed by the magnetic Compton scattering or (ii) the thermal radiation of deep atmospheric layers partly Comptonized in the overheated upper layers. In addition to GX 304-1 and X Persei, more examples of low-luminosity states need to be observed before the final conclusion on the physical mechanisms behind spectrum formation can be made.

In this Letter we consider one of the best studied Be/X-ray binaries, A $0535+262$, ideally suitable for such kind of studies. The source contains a slowly rotating NS $\left(P_{\text {spin }} \approx 104 \mathrm{~s} ; \quad\right.$ Rosenberg et al. 1975) orbiting around B0IIIe star HDE 245770 at distance around $2 \mathrm{kpc}$ from the Sun (Steele et al. 1998). A relatively long orbital period $P_{\text {orb }} \approx 110 \mathrm{~d}$ (Finger et al. 1996) implies that the source stays in the quiescence for a long period of time between periastron passages. Previously the source was observed in deep quiescence state with luminosity around or below $\sim 10^{34} \mathrm{erg} \mathrm{s}^{-1}$ several times with different instruments (Negueruela et al. 2000; Orlandini et al. 
Table 1. Observational log of A $0535+262$.

\begin{tabular}{lccc}
\hline ObsID & $\begin{array}{c}T_{\text {start }} \\
\text { MJD }\end{array}$ & $\begin{array}{c}T_{\text {stop }} \\
\text { MJD }\end{array}$ & $\begin{array}{c}\text { Exposure } \\
\text { ks }\end{array}$ \\
\hline \multicolumn{4}{c}{ NuSTAR observations } \\
80001016002 & 57064.17 & 57064.68 & 21.5 \\
80001016004 & 57067.07 & 57067.73 & 29.7 \\
90401370001 & 58478.11 & 58479.51 & 54.9 \\
\hline \multirow{5}{*}{ Swift observations } \\
00081432001 & 57064.17 & 57064.24 & 1.9 \\
00035066052 & 57068.89 & 57068.90 & 1.1 \\
00088834001 & 58479.60 & 58479.67 & 1.9 \\
\hline
\end{tabular}

2004; Rothschild et al. 2013; Doroshenko et al. 2014). It was demonstrated (see e.g. Doroshenko et al. 2014) that the emission even at such a low luminosity is dominated by the accretion from the "cold" accretion disc (Tsygankov et al. 2017). However, the low sensitivity of the available data above $10 \mathrm{keV}$ did not allow to study the broad band spectrum of A $0535+262$ at this luminosity. For this purpose we performed deep NuSTAR observations of A $0535+262$ between two type-I outbursts when the luminosity was as low as $\sim 7 \times 10^{34} \mathrm{erg} \mathrm{s}^{-1}$.

To determine the physical origin of the spectral components it is crucial to know the strength of the NS magnetic field. In the case of A $0535+262$ it is known from the cyclotron resonant scattering feature (CRSF) energy of $\sim 45$ $\mathrm{keV}$ and its first harmonic around $100 \mathrm{keV}$ (Kendziorra et al. 1994; Grove et al. 1995; Kretschmar et al. 1996). Unlike some others low-luminosity XRPs, A $0535+262$ exhibits no significant positive correlation of the cyclotron line energy with luminosity in the pulse-averaged spectra (Caballero et al. 2007, 2013; Ballhausen et al. 2017, however, see Sartore et al. 2015). At the same time such correlation was observed in the pulse-amplitude-resolved spectroscopy (Klochkov et al. 2011). Investigation of the cyclotron energy behaviour at very low luminosity is another topic we address below.

\section{DATA ANALYSIS}

In this work we use three NuSTAR (Harrison et al. 2013) observations of the source performed in wide range of luminosities. Two brighter observations were taken in the declining phase of the outburst in the beginning of 2015 (Ballhausen et al. 2017), and the third one - on 2018 December 26 (ObsID 90401370001), in a deep quiescent state, 3 months after the outburst that ended in October 2018.

The raw data were processed following the standard data reduction procedures described in the NuSTAR user guide, and using the standard NuSTAR Data Analysis Software NUSTARDAS v1.8.0 provided under HEASOFT V6.25 with the CALDB version 20181022. The source and background spectra were extracted from the circular regions with radii of $50^{\prime \prime}$ and $90^{\prime \prime}$, respectively, using the NUPRODUCTS routine. The background was extracted from a source-free region in the corner of the field of view. Final spectra were optimally rebinned using the prescription in Kaastra \& Bleeker (2016).

To expand our spectral analysis to the softer energy band, we also used the data from the XRT telescope
(Burrows et al. 2005) on-board the Neil Gehrels Swift Observatory (Gehrels et al. 2004) obtained simultaneously or very close in time to the NuSTAR observations. The observational log can be found in Table 1.

The spectral extraction from the XRT data was done using the online tools (Evans et al. 2009) ${ }^{1}$ provided by the UK Swift Science Data Centre. To fit the spectra in the XSPEC package we binned the spectra to have at least 1 count per energy bin and fitted them using W-statistic (Wachter et al. 1979). The data from Swift/XRT and NuSTAR were used in the $0.3-10 \mathrm{keV}$ and $3.5-79 \mathrm{keV}$ bands, respectively.

\section{RESULTS}

In all three $N U S T A R$ observations we detected strong pulsations from A $0535+262$ with the pulsed fraction decreasing from $\sim 50$ to $\sim 20$ per cent following the decrease of the flux. The pulse profile also evolves with luminosity becoming more single peaked at lower flux. In either case we did not detect significant phase lags between relatively soft (3$13 \mathrm{keV}$ ) and hard (13-79 keV) pulse profiles expected in the case of different geometrical structures responsible for emission in these energy bands.

In contrast to the timing properties, the source spectrum strongly depends on the accretion rate. Spectra in the two brighter observations were already discussed by Ballhausen et al. (2017). Particularly, they showed that the spectrum of the bright state at luminosity $2 \times 10^{36} \mathrm{erg} \mathrm{s}^{-1}$ can be fitted with a single either empirical or physically motivated continuum model. However, when the luminosity dropped down to $0.6 \times 10^{36} \mathrm{erg} \mathrm{s}^{-1}$, strong residuals started to appear around $30 \mathrm{keV}$, depending on the assumed continuum shape. The authors claimed that only PLCUT model was able to describe the data satisfactorily. However, an additional broad Gaussian emission component centered at $\sim 26 \mathrm{keV}$ was required. As we discuss below, this is not the only way to describe the broadband continuum, however, it illustrates nicely that some hard excess emission appears already at intermediate fluxes.

In our NuSTAR observation the source luminosity dropped further by an order of magnitude to $\sim 7 \times 10^{34}$ $\mathrm{erg} \mathrm{s}^{-1}$ and its spectrum changed dramatically (see Fig. 1). Following Tsygankov et al. (2019), and to facilitate comparison of the spectra in all three observations and between the sources, we fitted all three spectra with a twocomponent continuum consisting of two Comptonization components (СОМРTT) modified with the interstellar absorption (PHABS), fluorescent iron line (GAUSS) and cyclotron line with Gaussian profile (GABS). The final model is PHABS $\times($ GAUSS + COMTT + COMPTT $) \times$ GABS in XSPEC with best-fitting parameters presented in Table 2 . The temperature of the seed photons was tied for the low- and the highenergy components. The position and the width of the iron emission line was fixed at $6.4 \mathrm{keV}$ and $0.1 \mathrm{keV}$, respectively.

\footnotetext{
1 http://www.swift.ac.uk/user_objects/
} 
Table 2. Best-fitting results with PHABS(GAU+COMPTT+COMPTT)GABS model to the broad-band spectra of A $0535+262$ in different states obtained with NUSTAR and Swift/XRT.

\begin{tabular}{|c|c|c|c|c|c|c|}
\hline \multirow{2}{*}{$\begin{array}{l}\text { Parameter }^{a} \\
n_{\mathrm{H}}, 10^{22} \mathrm{~cm}^{-2}\end{array}$} & \multicolumn{2}{|c|}{ Low state } & \multicolumn{2}{|c|}{ Medium state } & \multicolumn{2}{|c|}{ High state } \\
\hline & \multicolumn{2}{|c|}{$0.08 \pm 0.07$} & \multicolumn{2}{|c|}{$0.06 \pm 0.05$} & \multicolumn{2}{|c|}{$0.08 \pm 0.02$} \\
\hline$T_{0}, \mathrm{keV}$ & \multicolumn{2}{|c|}{$0.79 \pm 0.05$} & \multicolumn{2}{|c|}{$0.80 \pm 0.02$} & \multicolumn{2}{|c|}{$0.74 \pm 0.01$} \\
\hline$T_{\mathrm{p}}, \mathrm{keV}^{b}$ & $2.4 \pm 0.3$ & $14.5 \pm 1.1$ & $2.9 \pm 0.3$ & $9.8 \pm 0.3$ & $2.4 \pm 0.4$ & $10.4 \pm 0.2$ \\
\hline$\tau_{\mathrm{p}}$ & $6.8 \pm 0.2$ & $>10$ & $7.4 \pm 0.2$ & $>10$ & $>10$ & $4.5 \pm 0.6$ \\
\hline$E_{\text {cyc }}, \mathrm{keV}$ & \multicolumn{2}{|c|}{$47.7 \pm 0.8$} & \multicolumn{2}{|c|}{$45.8 \pm 0.4$} & \multicolumn{2}{|c|}{$46.1 \pm 0.2$} \\
\hline$W_{\text {сус }}, \mathrm{keV}$ & \multicolumn{2}{|c|}{$12.6 \pm 1.5$} & \multicolumn{2}{|c|}{$9.6 \pm 0.5$} & \multicolumn{2}{|c|}{$11.3 \pm 0.3$} \\
\hline$\tau_{\text {cyc }}$ & \multicolumn{2}{|c|}{$2.4 \pm 0.5$} & \multicolumn{2}{|c|}{$1.1 \pm 0.1$} & \multicolumn{2}{|c|}{$0.94 \pm 0.03$} \\
\hline$F_{\mathrm{X}}, 10^{-10} \mathrm{erg} \mathrm{s}^{-1} \mathrm{~cm}^{-2}$ & \multicolumn{2}{|c|}{$1.4 \pm 0.2$} & \multicolumn{2}{|c|}{$13 \pm 3$} & \multicolumn{2}{|c|}{$36 \pm 5$} \\
\hline$\chi_{\text {red }}^{2}$ (d.o.f.) & \multicolumn{2}{|c|}{$0.96(431)$} & \multicolumn{2}{|c|}{$1.04(485)$} & \multicolumn{2}{|c|}{$1.17(511)$} \\
\hline
\end{tabular}

${ }^{a}$ Here $T_{\mathrm{p}}, \tau_{\mathrm{p}}$ and $T_{0}$ are the plasma temperature, plasma optical depth and temperature of the seed photons for the COMPTT model, respectively. Fluxes are given in the $0.5-100 \mathrm{keV}$ energy range. Parameters of the iron line, modelled with the Gaussian, were fixed at the energy of $E_{\mathrm{Fe}}=6.4 \mathrm{keV}$ and width of $0.1 \mathrm{keV}$.

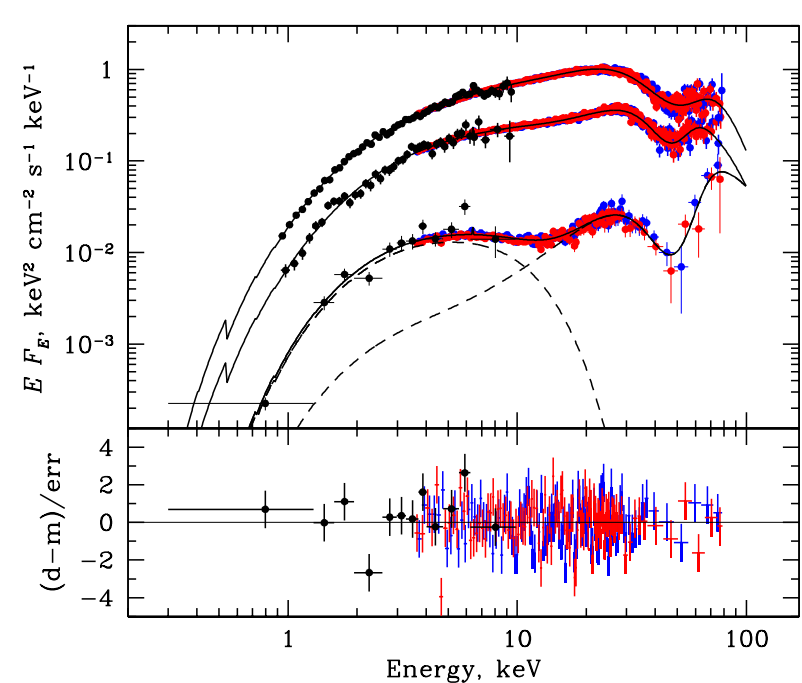

Figure 1. $E F_{\mathrm{E}}$ spectra of A $0535+262$ in different states with luminosity varying by almost two orders of magnitude, from $2 \times$ $10^{36}$ and $0.6 \times 10^{36} \mathrm{erg} \mathrm{s}^{-1}$ for the top two spectra down to $\sim$ $7 \times 10^{34} \mathrm{erg} \mathrm{s}^{-1}$ for the bottom one. The black, red and blue points correspond to the Swift/XRT and NuSTAR FPMA and FPMB data, respectively. The solid lines represent the best-fitting models listed in Table 2. Dashed lines represent two COMPTT components constituting the emission continuum. The corresponding residuals for the lowest luminosity state of A $0535+262$ are presented at the lower panel.

\section{DISCUSSION}

\subsection{Origin of the high-energy component}

The broadband spectra of the three X-ray pulsars observed so far in the low state presented in Fig. 2 show striking similarity. Particularly, in all three cases the spectrum can be described as a blend of two broad components peaking around 5-7 $\mathrm{keV}$ and $30-50 \mathrm{keV}$, respectively. We note that while the single component cutoff power law modified by a broad absorption feature at $\sim 30 \mathrm{keV}$ interpreted as a cyclotron line had been invoked previously to describe the spectrum

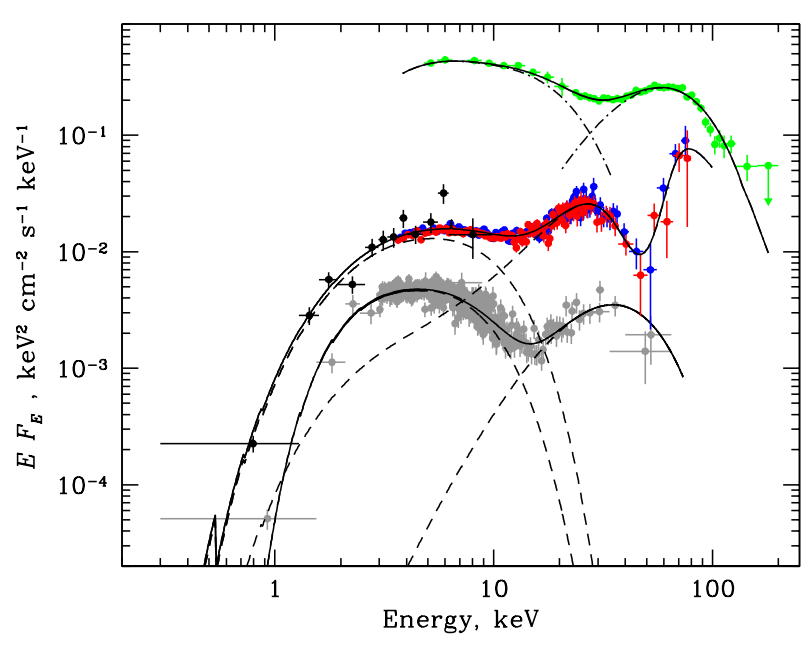

Figure 2. The spectra of $\mathrm{X}$ Persei (green points; Doroshenko et al. 2012), GX 304-1 (grey points; Tsygankov et al. 2019) and A $0535+262$ (this work) observed at low accretion rates, along with the best-fitting models consisting of two Comptonization components (black solid lines). Separate model components (COMPTT in XSPEC) are shown with dashed lines.

of X Persei (Coburn et al. 2001; Lutovinov et al. 2012), this approach actually appears to be unjustified both for X Persei (Doroshenko et al. 2012) and GX 304-1 (Tsygankov et al. 2019). It is even more true for A $0535+262$ where the cyclotron line is also observed on top of the hard component, which completely rules out such interpretation for this source. Given the overall similarity of spectra of the three sources, we conclude thus that the hard component is not artificially appearing because of the presence of a broad cyclotron line at intermediate energies, but rather represents a physically independent component.

The question is then, what is the origin of the two components. From observational point of view, both appear to be consistent with Comptonization by relatively cool $(k T \sim 2.5 \mathrm{keV})$ and hot $(k T \sim 15 \mathrm{keV})$ electrons. While the soft component is readily associated with thermal 
Comptonization within the accretion-heated hotspot, the origin of the hard component is less clear. As discussed by Tsygankov et al. (2019), bulk Comptonization of soft seed photons in the accretion channel suggested previously for X Persei (Doroshenko et al. 2012) is unlikely to be effective because of the extremely low optical depths in the accretion channel at the observed accretion rates. We note that this also likely applies to any kind of Comptonization of soft seed photons unless their source originates deep in the atmosphere of the neutron star.

Another interpretation discussed by Di Salvo et al. (1998) and Coburn et al. (2001) for X Persei is based on idea first suggested by Nelson et al. (1995). In this scenario fast ions of the accretion flow decelerate in the atmosphere mostly by collisional excitation of atmospheric electrons to the upper Landau levels. These then quickly recombine emitting photons at the cyclotron energy which propagate through the atmosphere losing the energy by the electron recoils, and ultimately produce a soft thermal continuum plus a hard non-thermal line just below the cyclotron energy. Coburn et al. (2001) dismissed this idea based on the fact that observed spectrum of X Persei is well described with a broad component rather than a comparatively narrow line with a sharp cutoff above the cyclotron energy predicted by Nelson et al. (1995). Doroshenko et al. (2012) also noted that luminosity of the hard component in X Persei, which constitutes $\sim 30$ per cent of the total luminosity is substantially higher than that predicted by Nelson et al. (1995), thus also rejecting this scenario based on energetic arguments. We note, however, that Nelson et al. (1995) considered cold atmosphere with the temperature negligible compared to the cyclotron energy. This simplifies the calculations but also amplifies energy losses of high energy photons as they diffuse through the atmosphere, and thus diminish the contribution of the hard component. The qualitative picture discussed below is essentially an extension of the scenario by Nelson et al. (1995) with the assumption of the low-temperature atmosphere dropped.

Indeed, to keep the atmosphere cool, the energy deposited by the collisional excitation of electrons into Landau levels must still escape from the emission region, predominantly through thermal free-free emission. However, based on the analogy with the accretion-heated magnetized and non-magnetized NS atmospheres (see, e.g. Zane et al. 2000; Deufel et al. 2001; Suleimanov et al. 2018; González-Caniulef et al. 2019, and discussion therein), the density in the upper atmosphere layers is likely insufficient to make this process effective. The upper atmosphere layers are thus expected to be overheated, and thermal electron energies may actually be comparable with the cyclotron energy. In this case the energy losses of resonant photons through recoil will be negligible, and we can anticipate a larger fraction of flux to escape around the cyclotron energy in a broad component with the width defined by the temperature of the atmosphere. We note increased temperature of the hard component at low luminosity (see Table 2), which is likely associated with the increased temperature of the upper atmosphere layers (Mushtukov et al., in preparation). A narrow absorbtion feature on top of this component may still be produced in the accretion flow just above the NS surface. Indeed, the Thomson optical thickness of the accretion channel at $L \sim 7 \times 10^{34} \mathrm{erg} \mathrm{s}^{-1}$ is $\tau_{\mathrm{T}} \sim 10^{-2}$, but the opti-

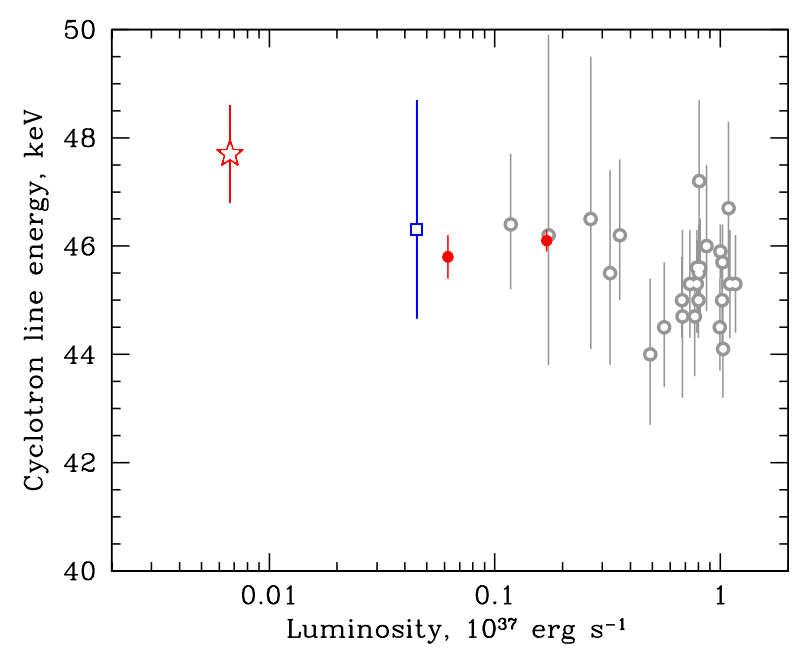

Figure 3. The dependence of the cyclotron line energy in A $0535+262$ on the bolometric $(0.5-100 \mathrm{keV})$ luminosity. The red star represents measurement in the lowest luminosity state, while the two filled red circles correspond to the brighter NuSTAR observations (see Table 2). The blue open square and grey open circles show measurements taken from Terada et al. (2006) and Caballero et al. (2007), respectively.

cal thickness to the resonant scattering is $\tau \gtrsim 10^{2}$, which is more than enough to explain the observed absorption feature around $47 \mathrm{keV}$.

It is important to emphasize that while the qualitative scenario outlined above has to be confirmed with detailed numerical computations, it appears that collisional excitation of electrons in strong magnetic field is the only way to deposit the power of the accretion flow to higher photon energies where a significant fraction of the observed flux emerges. The results presented above show that the presence of a hard component appears quite common in low-luminosity XRPs, therefore observing additional sources with a range of magnetic fields becomes now feasible.

\subsection{Cyclotron line luminosity dependence}

Depending on the local mass accretion rate onto the magnetic poles of the NS, all XRPs can be roughly divided in two groups with different response of the cyclotron energy on the changing luminosity. In bright sources with fluxes high enough to stop accreting material above the NS surface, a negative correlation of the cyclotron energy with luminosity is expected (Becker et al. 2012; Poutanen et al. 2013) and observed in at least one bright transient XRP V 0332+53 (Tsygankov et al. 2006). In the opposite case, when luminosity of the source is below the critical one $\left(L_{\text {crit }} \sim\right.$ $10^{37} \mathrm{erg} \mathrm{s}^{-1}$; see Basko \& Sunyaev 1976; Mushtukov et al. 2015a) a positive correlation is observed in several sources (Staubert et al. 2007; Mushtukov et al. 2015b, for more details, see a review by Staubert et al. 2019). Transition between these two regimes in $\mathrm{V} 0332+53$ was recently discovered by Doroshenko et al. (2017).

A $0535+262$ has a relatively low luminosity rarely exceeding $\sim 10^{37} \mathrm{erg} \mathrm{s}^{-1}$ even in the maximum of giant outbursts. Therefore, the source belongs to the sub-critical group of XRPs where positive correlation can be expected. 
During the last decade A $0535+262$ was studied in great details with different instruments in order to detect such a correlation. However, in the pulse-averaged data it was not found in very broad range of mass accretion rates (see, e.g. Caballero et al. 2007, 2013; Ballhausen et al. 2017).

Our NuSTAR observation allowed us to measure the cyclotron line energy in A $0535+262$ with high accuracy at luminosity almost one order of magnitude below the lowest available in the literature. The results shown in Fig. 3 confirm the absence of the positive correlation of the cyclotron energy on the mass accretion rate in the source. Instead of positive correlation, some hint to the negative correlation of the cyclotron energy on the source luminosity is seen at the lowest luminosity. However, it has a very low significance, especially taking into account substantial change in the shape of the underlying continuum which can affect the deduced line energy. We thus confirm earlier findings and conclude that there is no strong evidence for correlation or anti-correlation of the line energy with luminosity in this source.

\section{CONCLUSION}

In the work we presented results of the broadband spectral analysis of emission from the transient XRP A 0535+262, obtained for the first time with the NuSTAR observatory at the very low luminosity $\sim 7 \times 10^{34} \mathrm{erg} \mathrm{s}^{-1}$. The data revealed that the spectrum can be described as a blend of two broad components peaking around 5-7 keV and 30-50 $\mathrm{keV}$ and thus differs dramatically from the cutoff powerlaw spectrum observed at higher fluxes. We explain the appearance of the high-energy component by recombination of electrons collisionally excited to the upper Landau levels in the heated layers of the neutron star atmosphere. We were also able to accurately measure the cyclotron line energy $E_{\text {cyc }}=47.7 \pm 0.8 \mathrm{keV}$ at a low luminosity never achieved before by the broad-band instruments. This allowed us to firmly exclude a positive correlation of the cyclotron energy with the accretion rate in this source.

\section{ACKNOWLEDGEMENTS}

This work was supported by the Russian Science Foundation grant 19-12-00423. We also acknowledge the support from the Academy of Finland travel grants 317552, 324550 (SST) and 322779 (JP), the Väisälä Foundation (SST), the Netherlands Organization for Scientific Research Veni Fellowship (AAM), the DFG grant WE 1312/51-1 (VFS) and the German Academic Exchange Service (DAAD) travel grant 57405000 (VFS). We grateful to the NuSTAR team for approving the DDT observation of A $0535+262$.

\section{REFERENCES}

Ballhausen R., et al., 2017, A\&A, 608, A105

Basko M. M., Sunyaev R. A., 1976, MNRAS, 175, 395

Becker P. A., Wolff M. T., 2007, ApJ, 654, 435

Becker P. A., et al., 2012, A\&A, 544, A123

Burrows D. N., et al., 2005, Space Sci. Rev., 120, 165

Caballero I., et al., 2007, A\&A, 465, L21
Caballero I., et al., 2013, ApJ, 764, L23

Coburn W., Heindl W. A., Gruber D. E., Rothschild R. E., Staubert R., Wilms J., Kreykenbohm I., 2001, ApJ, 552, 738

Deufel B., Dullemond C. P., Spruit H. C., 2001, A\&A, 377, 955

Di Salvo T., Burderi L., Robba N. R., Guainazzi M., 1998, ApJ, 509,897

Doroshenko V., Santangelo A., Kreykenbohm I., Doroshenko R., 2012, A\&A, 540, L1

Doroshenko V., Santangelo A., Doroshenko R., Caballero I., Tsygankov S., Rothschild R., 2014, A\&A, 561, A96

Doroshenko V., Tsygankov S. S., Mushtukov A. A., Lutovinov A. A., Santangelo A., Suleimanov V. F., Poutanen J., 2017, MNRAS, 466, 2143

Evans P. A., et al., 2009, MNRAS, 397, 1177

Farinelli R., Ferrigno C., Bozzo E., Becker P. A., 2016, A\&A, 591, A29

Finger M. H., Wilson R. B., Harmon B. A., 1996, ApJ, 459, 288

Gehrels N., et al., 2004, ApJ, 611, 1005

González-Caniulef D., Zane S., Turolla R., Wu K., 2019, MNRAS, 483,599

Grove J. E., et al., 1995, ApJ, 438, L25

Harrison F. A., et al., 2013, ApJ, 770, 103

Kaastra J. S., Bleeker J. A. M., 2016, A\&A, 587, A151

Kendziorra E., et al., 1994, A\&A, 291, L31

Klochkov D., Staubert R., Santangelo A., Rothschild R. E., Ferrigno C., 2011, A\&A, 532, A126

Kretschmar P., et al., 1996, A\&AS, 120, 175

Lutovinov A., Tsygankov S., Chernyakova M., 2012, MNRAS, 423,1978

Mushtukov A. A., Suleimanov V. F., Tsygankov S. S., Poutanen J., 2015a, MNRAS, 447, 1847

Mushtukov A. A., Tsygankov S. S., Serber A. V., Suleimanov V. F., Poutanen J., 2015b, MNRAS, 454, 2714

Negueruela I., Reig P., Finger M. H., Roche P., 2000, A\&A, 356, 1003

Nelson R. W., Wang J. C. L., Salpeter E. E., Wasserman I., 1995, ApJ, 438, L99

Orlandini M., et al., 2004, Nuclear Physics B Proceedings Supplements, 132,476

Poutanen J., Mushtukov A. A., Suleimanov V. F., Tsygankov S. S., Nagirner D. I., Doroshenko V., Lutovinov A. A., 2013, ApJ, 777, 115

Rosenberg F. D., Eyles C. J., Skinner G. K., Willmore A. P., 1975, Nature, 256, 628

Rothschild R., et al., 2013, ApJ, 770, 19

Sartore N., Jourdain E., Roques J. P., 2015, ApJ, 806, 193

Staubert R., Shakura N. I., Postnov K., Wilms J., Rothschild R. E., Coburn W., Rodina L., Klochkov D., 2007, A\&A, 465, L25

Staubert R., et al., 2019, A\&A, 622, A61

Steele I. A., Negueruela I., Coe M. J., Roche P., 1998, MNRAS, 297, L5

Suleimanov V. F., Poutanen J., Werner K., 2018, A\&A, 619, A114

Terada Y., et al., 2006, ApJ, 648, L139

Tsygankov S. S., Lutovinov A. A., Churazov E. M., Sunyaev R. A., 2006, MNRAS, 371, 19

Tsygankov S. S., Mushtukov A. A., Suleimanov V. F., Doroshenko V., Abolmasov P. K., Lutovinov A. A., Poutanen J., 2017, A\&A, 608, A17

Tsygankov S. S., Rouco Escorial A., Suleimanov V. F., Mushtukov A. A., Doroshenko V., Lutovinov A. A., Wijnands R., Poutanen J., 2019, MNRAS, 483, L144

Wachter K., Leach R., Kellogg E., 1979, ApJ, 230, 274

Zane S., Turolla R., Treves A., 2000, ApJ, 537, 387

This paper has been typeset from a $\mathrm{T}_{\mathrm{E}} \mathrm{X} / \mathrm{LATEX}$ file prepared by the author. 